\title{
How successful are we in shoulder stabilization?
}

\author{
Roland Becker
}

Published online: 22 September 2012

(C) Springer-Verlag 2012

The shoulder is the most mobile joint in the human body, mainly due to minimal osseous constraint. Soft-tissue structures such as muscles, ligaments, tendons, and labrum are divided into active and passive stabilizers and are responsible for primary stability. The weakest part of the soft-tissue envelope around the shoulder is the anterior region, i.e., the rotator cuff interval located between the supraspinatus and subscapularis muscles. Shoulder dislocations occur in the anterior direction in $90 \%$ of all cases.

Unidirectional instability caused by trauma often requires surgery. However, we now have good knowledge that the risk of second dislocation decreases with increasing age. One may primarily consider nonsurgical treatment when the patient is $>30$ years of age [5]. Younger patients require early surgery to prevent redislocation and further damage to the joint.

Different pathologies are found after acute shoulder dislocation, such as labral tears, anterior capsular laxity, and glenohumeral ligament insufficiency. Also, superior labral tear, rotator cuff tear, Hill-Sachs fracture, or anterior glenoid fracture are commonly seen [4]. These pathological findings are probably more common than previously anticipated and need to be addressed during surgery in order to achieve the best possible clinical outcome. However, the redislocation rate after shoulder stabilization surgery shows a range between $6 \%$ and $30 \%$. The question remains: why does the failure rate differ so much between studies?

\footnotetext{
R. Becker $(\bowtie)$

Department of Orthopaedic and Trauma Surgery,

Hospital Brandenburg, Hochstrasse 26,

14770 Brandenburg, Havel, Germany

e-mail: roland_becker@yahoo.de
}

Several factors may cause failure after shoulder stabilization surgery. These factors are partly patient related (age, sex, shoulder anatomy, degree of joint laxity, sports activity level and type, number of previous dislocations, dominant side, compliance, and reinjury), partly surgery related (surgical technique), and partly related to the pathology itself [bony or soft-tissue injury of the anterior part of the shoulder, and combined injuries, such as superior labral anterior and posterior (SLAP) lesions or rotator cuff tear]. A multitude of clinical studies are available in the literature focusing on outcome after shoulder stabilization. However, it is still difficult to take all factors into account when analyzing the patient-related outcome. It is even more difficult to weight between these factors. Most of the time there is a lack of information about the important predisposing factors of shoulder instability in order to be able to analyze in detail the reason for failure.

A review of the literature might help provide useful information with regards to the indication for nonsurgical or surgical management, timing of shoulder repair, and to clarify which surgical procedure might be the most appropriate. The lead article of this issue of the journal focuses on risk factors that might cause failure after Bankart repair and summarizes the articles published in peer-reviewed journals over the last 10 years [7]. Interesting findings are reported, but most of these studies present level IV evidence. Patient age, gender, and dislocation number appear to have a significant impact on outcome. For instance: why do men tend to experience shoulder dislocation more frequently than women? One may expect more dislocations in women due to the natural joint laxity. Anatomical studies of the glenoid show differences in shape and height to width ratio between genders [6]. However, the impact of those differences on the incidence of shoulder dislocation remains unclear. 
Surgical technique influences clinical outcome. There is an ongoing discussion about mini-open or arthroscopic repair. A recent review of the literature showed better outcome after mini-open repair. In conclusion, further improvement of arthroscopic surgery is required instead of first recommending mini-open repair. There are many advantages of using arthroscopic techniques, such as minimizing surgical trauma, less pain after surgery, addressing additional pathologies, and-not to be forgotten-cosmetic aspects. Interestingly, Randelli et al. [7] found that using less than three anchors for stabilization showed significant increase in recurrence rate. Four or more anchors appear to be even safer in terms of lower redislocation rate. The price to achieve increased joint stability by using more anchors appears to be increased risk of osteoarthritis [3]. Recent results have shown, that the number of anchors and the state of the labrum appear to be the most important risk factors in terms of glenohumeral osteoarthritis.

Possibly the additional shift of the anterior capsule might be of help. Robotic testing of human specimens show that capsular shift decreases humeral-head external rotation but does not influence anterior translation [2]. The decrease in external rotation might prevent redislocation but, again, there is an increased risk of osteoarthritis. The site of capsular shift might be of major relevance. Nonanatomic repair most probably increases the risk of osteoarthritis due to alteration in joint biomechanics [1]. The anteroinferior shift of the capsule prevents reduction in external rotation in comparison with the anterior tightening of the capsule [9]. Sufficient shifting of the capsule is probably always needed when shoulder stabilization is performed. However, it is very difficult to quantify the amount of capsular shift necessary to achieve permanent successful shoulder stability in the long term without the increased risk of early osteoarthritis.

Better understanding of shoulder pathology and the development of new surgical techniques are major challenges. The continuous improvement in arthroscopic surgery and the development of new devices for fixation should have a positive impact on success rate. For instance, the change from a two-portal to a three-portal technique using the suprabicipital portal for the arthroscope allows better visualization of the anterior and inferior aspect of the neck of glenoid, allowing better preparation and fixation of the labrum capsule complex.
These are only a few examples to show the complexity of treating shoulder instability. Numerous factors may have a significant impact on shoulder-joint homeostasis. When surgery is required, a balance must be found between treating the pathology and subsequent damage, which might be caused to the joint at the time of surgery. Despite the known failure rates, the vast majority of patients show significant improvement after shoulder stabilization, with a good possibility of returning to their previous level of activity [8].

Knowledge of the patient's risk factors for recurring shoulder instability after repair, as analyzed by Randelli et al. [7] , helps us to better understand the pathology and tends to favor a more individual planning for patient management after traumatic shoulder dislocation.

\section{References}

1. Ahmad CS, Wang VM, Sugalski MT, Levine WN, Bigliani LU (2005) Biomechanics of shoulder capsulorrhaphy procedures. J Shoulder Elbow Surg 14:12S-18S

2. Bohnsack M, Bartels B, Ostermeier S, Rühmann O, Wellmann M, Mansouri F, Hurschler C (2009) Biomechanical stability of an arthroscopic anterior capsular shift and suture anchor repair in anterior shoulder instability: a human cadaveric shoulder model. Knee Surg Sports Traumatol Arthrosc 17:1493-1499

3. Franceschi F, Papalia R, Del Buono A, Vasta S, Maffulli N, Denaro V (2011) Glenohumeral osteoarthritis after arthroscopic Bankart repair for anterior instability. Am J Sports Med 39:1653-1659

4. Hintermann B, Gächter A (1995) Arthroscopic findings after shoulder dislocation. Am J Sports Med 23:545-551

5. Kandziora F, Jäger A, Bischof F, Herresthal J, Starker M, Mittlmeier T (2000) Arthroscopic labrum refixation for posttraumatic anterior shoulder instability: suture anchor versus transglenoid fixation technique. Arthroscopy 16:359-366

6. Merrill A, Guzman K, Miller SL (2009) Gender differences in glenoid anatomy: an anatomic study. Surg Radiol Anat 31:183-189

7. Randelli P, Ragone V, Carminati S, Cabitza P (2012) Risk factors for recurrence after Bankart repair a systematic review. Knee Surg Sports Traumatol Arthrosc. doi:10.1007/s00167-012-2140-1

8. Stein T, Linke RD, Buckup J, Efe T, von Eisenhart-Rothe R, Hoffmann R, Jäger A, Welsch F (2011) Shoulder sport-specific impairments after arthroscopic Bankart repair: a prospective longitudinal assessment. Am J Sports Med 39:2404-2414

9. Wang VM, Sugalski MT, Levine WN, Pawluk RJ, Mow VC, Bigliani LU (2005) Comparison of glenohumeral mechanics following a capsular shift and anterior tightening. J Bone Joint Surg Am 87:1312-1322 